Delft University of Technology

\title{
A 77/79-GHz Frequency Generator in 16-nm CMOS for FMCW Radar Applications Based on a 26-GHz Oscillator with Co-Generated Third Harmonic
}

Kuo, Feng Wei; Zong, Zhirui; Chen, Huan Neng Ron; Cho, Lan Chou; Jou, Chewn Pu; Chen, Mark; Staszewski, Robert Bogdan

DOI

10.1109/ESSCIRC.2019.8902490

Publication date

2019

Document Version

Accepted author manuscript

Published in

ESSCIRC 2019 - IEEE 45th European Solid State Circuits Conference

\section{Citation (APA)}

Kuo, F. W., Zong, Z., Chen, H. N. R., Cho, L. C., Jou, C. P., Chen, M., \& Staszewski, R. B. (2019). A 77/79$\mathrm{GHz}$ Frequency Generator in 16-nm CMOS for FMCW Radar Applications Based on a 26-GHz Oscillator with Co-Generated Third Harmonic. In ESSCIRC 2019 - IEEE 45th European Solid State Circuits Conference (pp. 53-56). [8902490] Institute of Electrical and Electronics Engineers (IEEE). https://doi.org/10.1109/ESSCIRC.2019.8902490

Important note

To cite this publication, please use the final published version (if applicable).

Please check the document version above. 


\title{
A 77/79-GHz Frequency Generator in 16-nm CMOS for FMCW Radar Applications Based on a 26-GHz Oscillator with Co-Generated Third Harmonic
}

\author{
Feng-Wei Kuo ${ }^{1}$, Zhirui Zong ${ }^{2}$, Huan-Neng Ron Chen ${ }^{1}$, Lan-Chou Cho ${ }^{1}$, Chewn-Pu Jou ${ }^{1}$, \\ Mark Chen ${ }^{1}$, Robert Bogdan Staszewski ${ }^{3,2}$ \\ ${ }^{1}$ Taiwan Semiconductor Manufacturing Company, Hsinchu, Taiwan. \\ ${ }^{2}$ Delft University of Technology, Delft, The Netherlands. $\quad{ }^{3}$ University College Dublin, Dublin 4, Ireland.
}

\begin{abstract}
This paper presents a digitally controlled frequency generator for dual frequency-band radar system that is optimized for $16 \mathrm{~nm}$ FinFET CMOS. It is based on a $21 \%$ wide tuning range, fine-resolution DCO with only switchable metal capacitors. A third-harmonic boosting DCO simultaneously generates 22.5 $28 \mathrm{GHz}$ and sufficiently strong $68-84 \mathrm{GHz}$ signals to satisfy shortrange radar (SRR) and medium/long range $\operatorname{radar}(\mathrm{M} / \mathrm{LRR})$ requirements. The $20.2 \mathrm{~mW}$ DCO emits $-97 \mathrm{dBc} / \mathrm{Hz}$ at $1 \mathrm{MHz}$ offset from $77 \mathrm{GHz}$, while fully satisfying metal density rules. It occupies $0.07 \mathrm{~mm}^{2}$, thus demonstrating both $43 \%$ power and $47 \%$ area reductions. The phase noise and $\mathrm{FoM}_{\mathrm{T}}$ (figure-ofmerit with tuning range) are improved by $1.8 \mathrm{~dB}$ and $0.2 \mathrm{~dB}$, respectively, compared to state-of-the-art.

Index Terms-Phase noise, DCO, oscillator, harmonic boost, common mode, FMCW, tuning range, automotive radar, $77 \mathrm{GHz}$.
\end{abstract}

\section{INTRODUCTION}

With the latest promises of autonomously driven vehicles, automotive radar applications expect great commercial interests. Among the advanced radar systems, the E-band frequency range $(76 \mathrm{GHz}$ to $81 \mathrm{GHz})$ is the most commonly used. To achieve ubiquitous sensing with fine resolution in short/medium/long range radar (SRR/MRR/LRR) applications, high signal-to-noise ratio (SNR) and large chirp bandwidth are necessary. The demands they place on monolithic frequency synthesizers are particularly tough, especially on low power consumption, low phase noise (PN) and wide tuning range (TR) with good linearity.

Oscillators and high-frequency dividers are the key challenges in the radar PLL design. A 77/79-GHz PLL architecture employs a mm-wave oscillator, which feeds both a mm-wave frequency divider back for a phase detection with a frequency reference clock, and a power amplifier (PA) to drive an antenna [1]. The oscillator PN is severely affected by a poor Q-factor of the resonant tank at $77 \mathrm{GHz}$. The dividers are typically power hungry and occupy large silicon area, and suffer from limited locking range. The frequency doubler/tripling PLL [2][3] relieves the aforementioned design challenges but shifts them to the frequency doubler or injection-locked frequency trippler (ILFT), so the solution-level issues remain.

To alleviate the above challenges of radar oscillators and dividers with minimum impact on other circuitry, a mm-wave radar LO with an implicit $3 \times$ multiplier is proposed here.

\section{CAnCEllation of Fundamental of Oscillator}

The basic concept of the presented idea (continuation of our earlier work for $60 \mathrm{GHz}$ [8] [9]) is that the oscillator

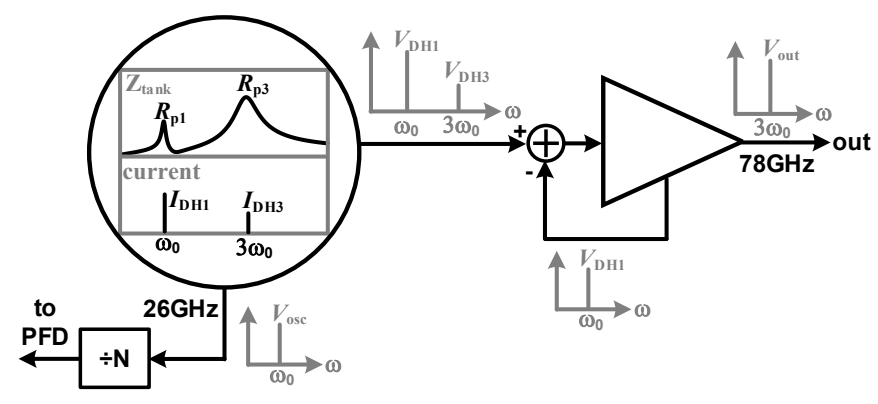

Fig. 1. Working principle of the proposed $77 / 79 \mathrm{GHz}$ frequency generator with the sub-harmonic soft-cancellation buffer.

simultaneously generates the fundamental at $22.5-28 \mathrm{GHz}$ and its 3rd harmonic at $68-84 \mathrm{GHz}$. The E-band radar carrier is fed forward to the buffer/PA, while the $23-28 \mathrm{GHz}$ signal is fed back for phase detection (e.g. TDC in an ADPLL). Consequently, the implicit $3 \times$ divider functionality is inherent with the oscillator thus avoiding any physical divider operating at the E-band carrier. This leads to a dramatic increase of the system-level efficiency.

However, the $\sim 26 \mathrm{GHz}$ fundamental harmonic must be suppressed in the buffer that follows the PLL. Otherwise, it can degrade the blocker tolerance of the radar receiver or even violate the emission mask in the transmitter. Hence, the feedforward path should exhibit strong suppression of the $26 \mathrm{GHz}$ tone. In this work, a soft cancellation technique of the $26 \mathrm{GHz}$ component is employed. Effectively, the buffer/PA does not actively respond to the $26 \mathrm{GHz}$ component input. It rejects the $26 \mathrm{GHz}$ fundamental by not providing a transconductance gain at this frequency.

For the PLL to cover the overall SRR/MRR/LRR radar requirements $(24-28 \mathrm{GHz}, 75-81 \mathrm{GHz})$, the DCO core should support a wide TR. Therefore, there are 5-bit binary PVT switched capacitors for coarse tuning, and 32-bit unary MSB and 64-bit unary LSB switched capacitors for fine tuning. This ensures the DCO core can cover full E-band frequency range with acceptable PN performance. Moreover, in the ADPLL design for radar applications, the strictest requirements are PN and power consumption. Thus, the DCO design is very critical since it dominates the ADPLL out-of-bandwidth performance.

\section{CiRCUit IMPlEMENTATiON}

The schematic and layout of the DCO are shown in Fig. 2(a)(b). To meet the strict PN and wide TR requirements, 


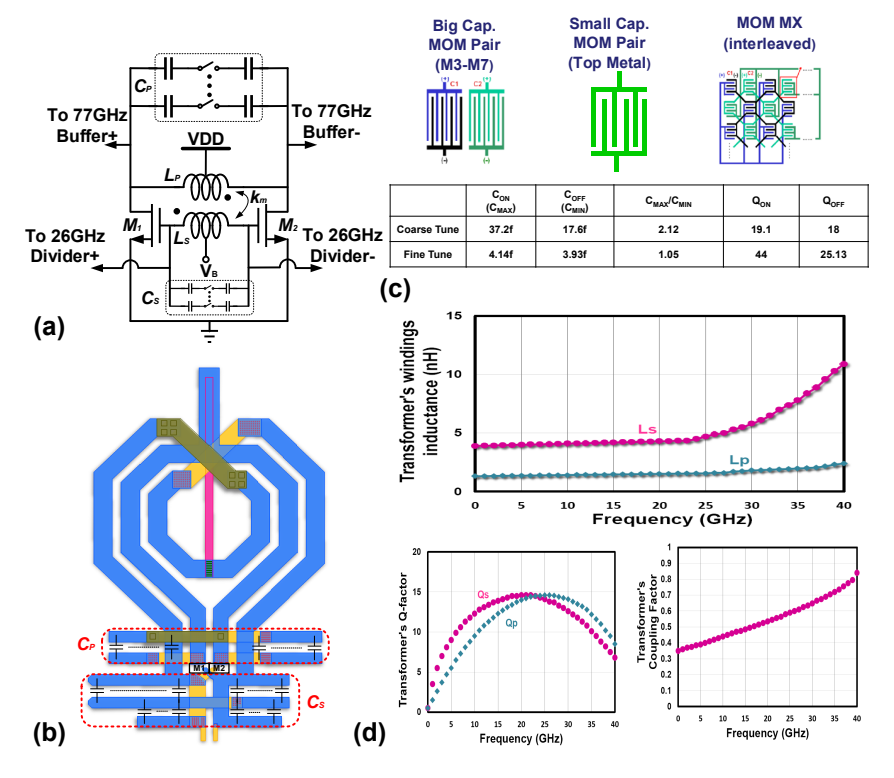

Fig. 2. (a) Schematic of the $77 / 79 \mathrm{GHz}$ DCO; (b) Concept layout of the DCO; (c)(d) single/multi-layer MoM capacitors and transformer effects with dummy metal fills on the $16 \mathrm{~nm}$ FinFET CMOS.

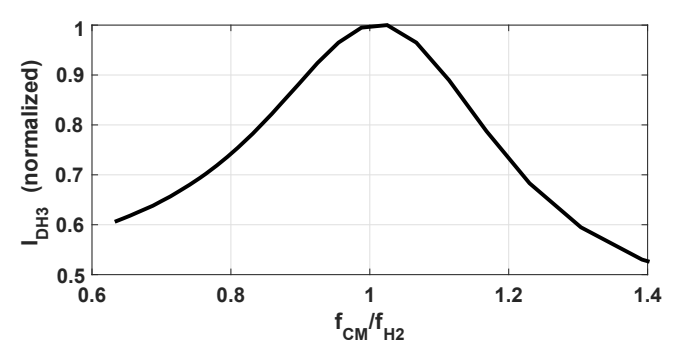

Fig. 3. Dependency of the $\mathrm{H} 3$ current in DCO on the CM resonant frequency. $\mathrm{CM}$ resonance at the second harmonic increases the third harmonic content.

it is of utmost importance to optimize the quality (Q) factors of transformer and switched capacitors.

In this design, several new process and circuit techniques are exploited to enhance the PN: First, porting of the oscillator design from 40-nm \& 28-nm CMOS ([8] [9]) to 16-nm FinFET CMOS has contributed to a lower power consumption and faster speed [10]. Second, use of custom single/multiple-layer MOM capacitors and a transformer with interleaved routing has reduced parasitic capacitance and mismatch, thus obtaining a better Q factor, as shown in Fig. 2(c)(d). Third, the DCO circuitry undergoes a large signal operation. A current richer (due to higher $g_{m}$ ) in harmonics is generated in the $\mathrm{g}_{m}$-devices $\left(M_{1}\right.$ and $\left.M_{2}\right)$. The $3 \mathrm{rd}$ harmonic (H3) current in the $26 \mathrm{GHz}$ $\mathrm{DCO}$ is reused for implicit frequency tripling. Leveraging the readily existing harmonic current in the DCO for frequency multiplication improves the power efficiency. In order to preserve the 3rd harmonic inside the oscillator, a transformerbased higher-order LC tank is employed to create an ancillary resonance at $\mathrm{H} 3$. To achieve a large $\mathrm{H} 3$ voltage swing in the DCO, a relatively small magnetic coupling coefficient $\left(k_{m}\right)$ of 0.6 is applied to achieve large tank impedance at this ancillary resonance.

To further increase the $\mathrm{H} 3$ swing, the $\mathrm{H} 3$ current is also boosted by enhancing the harmonic mixing effects in the DCO.
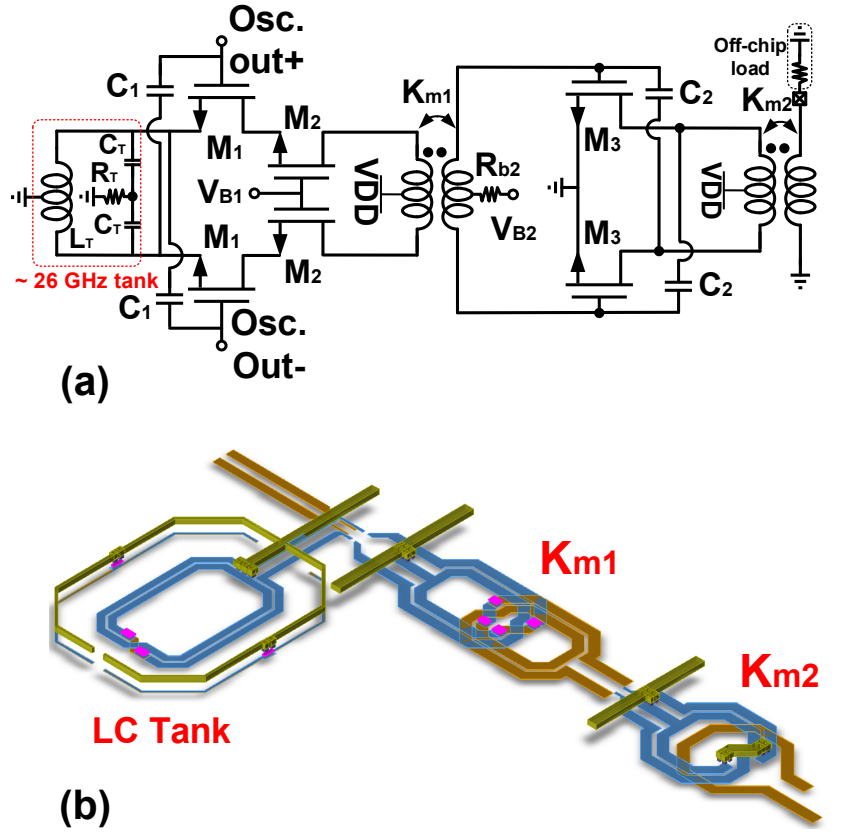

Fig. 4. (a) Schematic of $77 / 79 \mathrm{GHz}$ buffer with $26 \mathrm{GHz}$ suppression; (b) Concept layout of buffer.

The common-mode (CM) impedance of the LC tank affects the level of $\mathrm{H} 3$ current. Fig. 3 shows the dependency of the simulated $\mathrm{H} 3$ current on the $\mathrm{CM}$ resonant frequency $\left(f_{C M}\right)$. As we can see, the $\mathrm{H} 3$ current is maximized when $f_{C M}$ equals to the 2 nd harmonic $(\mathrm{H} 2)$ frequency $\left(f_{H_{2}}\right)$. Therefore, the CM impedance of the LC tank is tuned to resonate at $f_{H 2}$ by properly segmenting the differential and single-ended tank capacitance. In this way, the $\mathrm{H} 2$ voltage swing, which is preserved by the $\mathrm{H} 2$ resonance, mixes with the fundamental voltage component in the negative $\mathrm{g}_{m}$-devices of the DCO and delivers more $\mathrm{H} 3$ current. This technique can also improve the $1 / f^{3}$ noise corner. Simulations show that the $\mathrm{H} 3$ voltage swing is $40 \%$ of the fundamental in the oscillator. It relaxes the requirement on the following DCO buffer.

Both the fundamental and $\mathrm{H} 3$ components in the DCO are fed forward to the buffer stage. Ideally, the buffer would only react to the $\mathrm{H} 3$ component, while discarding the fundamental component. To achieve this goal, a fundamental component cancellation technique is implemented in this design, as shown in Fig. 4. An LC tank is connected to the sources of the input devices $\left(M_{1}\right)$. It exhibits a large impedance at the fundamental by means of parallel resonance at this frequency, while providing a very low impedance at $\mathrm{H} 3$. Therefore, the buffer acts as a common-source amplifier at H3, but with heavy degeneration at the fundamental. In other words, $M_{1}$ senses only the H3 component between its gate and source (i.e., $\left.v_{g s}\right)$. The fundamental component is therefore rejected. It also provides more gain to the signal of interest at $77 \mathrm{GHz}$. It is well known that a large blocker signal can desensitize the low-noise amplifiers (LNAs) in the receivers. The large $26 \mathrm{GHz}$ tone herein could also desensitize the conventional $77 \mathrm{GHz}$ buffers in a similar way. Since the $26 \mathrm{GHz}$ 'blocker' is rejected before reaching the input transistors of the proposed $77 \mathrm{GHz}$ buffer, it will not degrade the $77 \mathrm{GHz}$ signal gain. As 


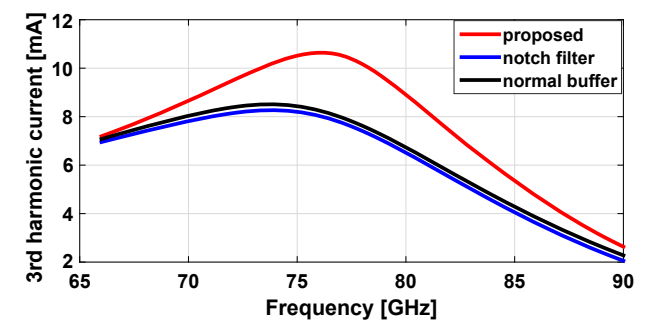

(a)

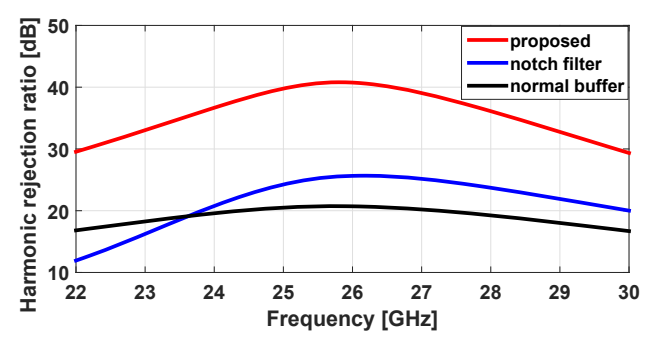

(b)

Fig. 5. Comparison of (a) the output current at $77 \mathrm{GHz}$ band and (b) harmonic rejection ratio at $26 \mathrm{GHz}$ band in different buffers.

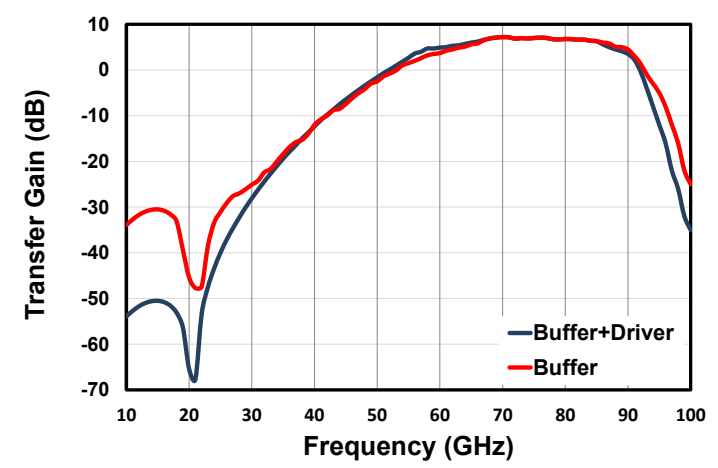

Fig. 6. Simulated transfer curves of the buffer stage and output driver.

shown in Fig. 5, the proposed buffer delivers the largest output current at $77 \mathrm{GHz}$ band and achieves the highest harmonic rejection ratio at $26 \mathrm{GHz}$ when compared to the notch filter and conventional buffers. The reduction of the fundamental component in $v_{g s}$ of $M_{1}$ also decreases the $\mathrm{H} 2$ component at the buffer output, which can result from the nonlinear distortion of the fundamental tone or harmonic mixing effects. One extra output driver stage is added in this design to drive the external $50 \Omega$ load and to deliver sufficient output power. Low $k_{m}$ coefficient $\left(k_{m 1}=0.25\right)$ is used to achieve wideband interstage matching. Fig. 6 shows the simulated transfer curve of the buffer stage and output driver.

\section{Measurement Results}

The proposed $77 / 79 \mathrm{GHz}$ frequency generator is prototyped in $16 \mathrm{~nm}$ FinFET CMOS. Fig. 10 shows the chip micrograph. It occupies a core area of $0.07 \mathrm{~mm}^{2}$. The phase noise (PN) and spectra of the generated E-band carrier are directly probed. $3 \mathrm{GHz}$ outputs of the frequency dividers can also be conveniently monitored.

The $77 / 79 \mathrm{GHz}$ DCO performance was measured using R\&S FSW signal and spectrum analyzers with an external harmonic mixer (up to $90 \mathrm{GHz}$ ). The tuning range (TR) of

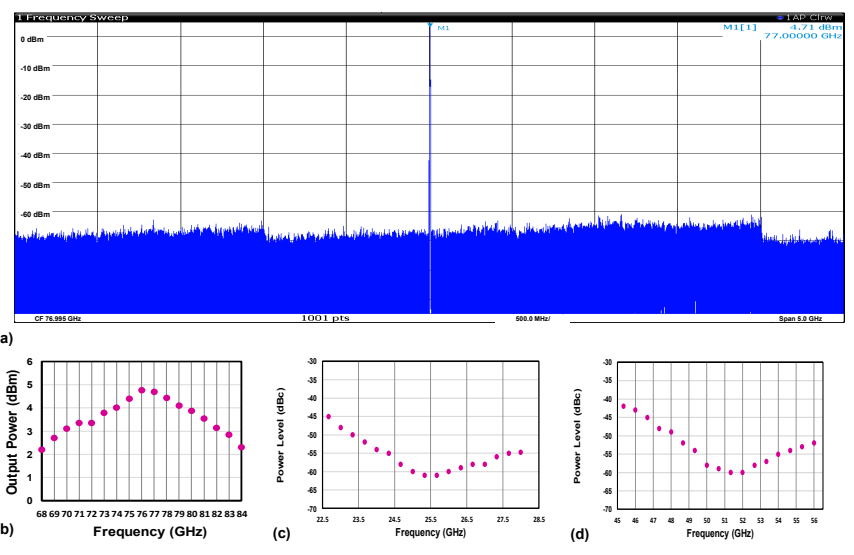

Fig. 7. Measured spectra at: (a)(b) $77 \mathrm{GHz}$ with sweep of the band, (c) 25.6 GHz with the band sweep; and (d) $51.3 \mathrm{GHz}$ with the band sweep.

the DCO is $68-84 \mathrm{GHz}(21 \%)$, covering all radar channels with sufficient margin. The oscillator core consumes $10 \mathrm{~mW}$ from a $0.85 \mathrm{~V}$ supply. In this design, the $77 / 79 \mathrm{GHz}$ buffer and $50-\Omega$ load driver stage deliver a maximum of $+6 \mathrm{dBm}$ power while consuming 10.2 and $15 \mathrm{~mW}$, respectively. The measured leakage of the $\sim 25.6 \mathrm{GHz}$ fundamental is $-61 \mathrm{dBc}$. Due to the common-mode leakage from the oscillator and nonlinearity of the driver, the second harmonic at $51.3 \mathrm{GHz}$ is visible at $-60 \mathrm{dBc}$. The leakage power level of the fundamental and second/third-harmonic tone at the output across the TR varies only $16 \mathrm{~dB}, 18 \mathrm{~dB}$ and $2.57 \mathrm{~dB}$, as shown in Fig. 7. The fundamental and second/third-harmonic power levels satisfy the out-of-band emission mask with sufficient margin.

The PN of the free-running $77 / 79 \mathrm{GHz}$ DCO are measured after the on-chip $\div 8$ frequency dividers and normalized to the $77 \mathrm{GHz}$ carrier, as shown in Fig. 8. A record-low $1 / f^{3}$ corner of $200 \mathrm{kHz}$ for an automotive radar oscillator is observed. The PN referred to the $77 \mathrm{GHz}$ and $3.2 \mathrm{GHz}$ carriers are $97 \mathrm{dBc} / \mathrm{Hz},-125 \mathrm{dBc} / \mathrm{Hz}$ at $1 \mathrm{MHz}$ offset, respectively.

Fig. 9 shows the $\mathrm{PN}$ at $1 \mathrm{MHz}$ offset and the corresponding FoM across the $21 \%$ TR. The PN varies between -96 and $-97.5 \mathrm{dBc} / \mathrm{Hz}$. The corresponding FoM changes between 180.4 and $181.8 \mathrm{dBc} / \mathrm{Hz}$ across the frequency range. Since the switched capacitors have lower Q-factor in the on-state, the FoM at lower frequencies decreases.

The performance of the proposed LO is summarized and compared to state-of-the-art $77 / 79 \mathrm{GHz}$ and mm-wave oscillators in Table I. The FoM and FoM ${ }_{\mathrm{T}}$ are $181.6 \mathrm{~dB}$ and $188.1 \mathrm{~dB}$ at $1 \mathrm{MHz}$ offset, respectively. The FoM is $2 \mathrm{~dB}$ better than the prior record in [8] [9] with different CMOS process nodes and frequency band.

\section{CONCLUSION}

We presented a $77 / 79-\mathrm{GHz}$ frequency generator implemented in 16-nm FinFET CMOS intended for automotive radar applications. It consists of a proposed third-harmonic digitally controlled oscillator (DCO) with a buffer rejecting its fundamental. It further utilizes custom single layer/multilayer MoM switched-capacitors to achieve good matching, high quality factor and wide tuning range. The DCO and buffer core occupy $0.07 \mathrm{~mm}^{2}$, while consuming $20.2 \mathrm{~mW}$ from a $0.85-\mathrm{V}$ supply. The $\mathrm{DCO}$ satisfies the $-97 \mathrm{dBc} / \mathrm{Hz} \mathrm{PN}$ at $1 \mathrm{MHz}$ offset over the full E-band range. 
TABLE I

PERFORMANCE COMPARISON WITH STATE-OF-THE-ART 77/79 GHZ OUTPUT OSCILLATOR SYSTEMS AND OUR PRIOR WORK IN OLDER TECHNOLOGY [8].

\begin{tabular}{|c|c|c|c|c|c|c|c|}
\hline & \multirow{2}{*}{ This work } & JSSC'18 & JSSC'18 & JSSC'18 & TMTT'13 & JSSC'16 \\
\hline & & & [3] & [4] & [5] & [6] & [8] \\
\hline \multicolumn{2}{|c|}{ CMOS Technology } & 16nm FinFET & $65 \mathrm{~nm}$ & $65 \mathrm{~nm}$ & $\begin{array}{l}\text { 130-nm } \\
\text { BiCMOS }\end{array}$ & $65 \mathrm{~nm}$ & $40 \mathrm{~nm}$ \\
\hline \multicolumn{2}{|c|}{ Supply Voltage (V) } & 0.85 & $1 / 2.5$ & 1 & $1.3-2.7$ & 1.2 & $0.7 / 1$ \\
\hline \multicolumn{2}{|c|}{ Type } & $\begin{array}{c}\text { HM extract } \\
\text { \& cancellation }\end{array}$ & Freq. doubler & fundamental & $\begin{array}{l}\text { frequency } \\
\text { multiplier }\end{array}$ & fundamental & $\begin{array}{c}\mathrm{HM} \\
\text { extract }\end{array}$ \\
\hline \multicolumn{2}{|c|}{$\begin{array}{c}\text { Tuning Range } \\
\text { (GHz) }\end{array}$} & $\begin{array}{l}68-84 \\
(21 \%)\end{array}$ & $\begin{array}{c}77-78.83 \\
(2.3 \%)\end{array}$ & $\begin{array}{c}67.8-81.4 \\
(18.2 \%)\end{array}$ & $\begin{array}{c}75-83 \\
(10.1 \%)\end{array}$ & $\begin{array}{c}75-78 \\
(15.5 \%)\end{array}$ & $\begin{array}{c}48.4-62.5 \\
(25.4 \%)\end{array}$ \\
\hline \multirow{2}{*}{$\begin{array}{c}\mathrm{PN} \\
(\mathrm{dBc} / \mathrm{Hz})\end{array}$} & $1 \mathrm{MHz}$ & -97 & -81.7 & -96 & -97 & -85.1 & -100.1 \\
\hline & $10 \mathrm{MHz}$ & -117.2 & NA & -116.5 & -120 & $-110^{\star *}$ & -122.3 \\
\hline \multirow{2}{*}{$\begin{array}{c}\text { FoM } \\
(\mathrm{dBc} / \mathrm{Hz})\end{array}$} & $1 \mathrm{MHz}$ & 181.6 & 165.1 & 179.7 & 168.2 & 170.6 & 179.8 \\
\hline & $10 \mathrm{MHz}$ & 181.8 & NA & 180 & 171.2 & 175.5 & 182 \\
\hline \multirow{2}{*}{$\begin{array}{c}\text { FoMt } \\
\text { (dBc/Hz) }\end{array}$} & $1 \mathrm{MHz}$ & 188.1 & 152.4 & 174.5 & 168.3 & 170.7 & 187.9 \\
\hline & $10 \mathrm{MHz}$ & 188.3 & NA & 175 & 171.3 & 175.6 & 190.1 \\
\hline \multirow{3}{*}{$\begin{array}{l}\text { Power } \\
(\mathrm{mW})\end{array}$} & Osc. & 10 & \multirow{2}{*}{26.8} & \multirow{2}{*}{25} & \multirow{2}{*}{$470^{* *}$} & 6.8 & 13.5 \\
\hline & Buf. & 10.2 & & & & 9.6 & 22 \\
\hline & Divider & $2.47^{*}$ & NA & NA & NA & 10.8 & NA \\
\hline \multicolumn{2}{|c|}{ Active Area $\left(\mathrm{mm}^{2}\right)$} & 0.07 & 0.2 & 0.06 & $0.72^{* *}$ & $0.09^{* *}$ & 0.1316 \\
\hline
\end{tabular}

* Power consumption of the $\sim 25 \mathrm{GHz}$ frequency divider. ${ }^{* *}$ graphically estimated.

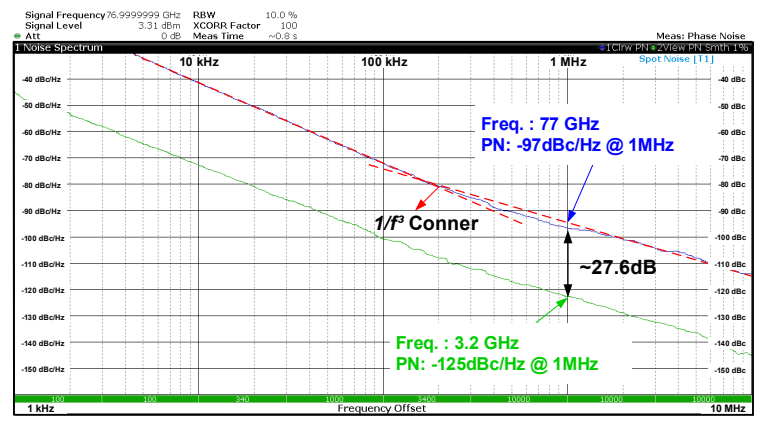

Fig. 8. Measured phase noise plots at $77 \mathrm{GHz}$ and $3.2 \mathrm{GHz}$.

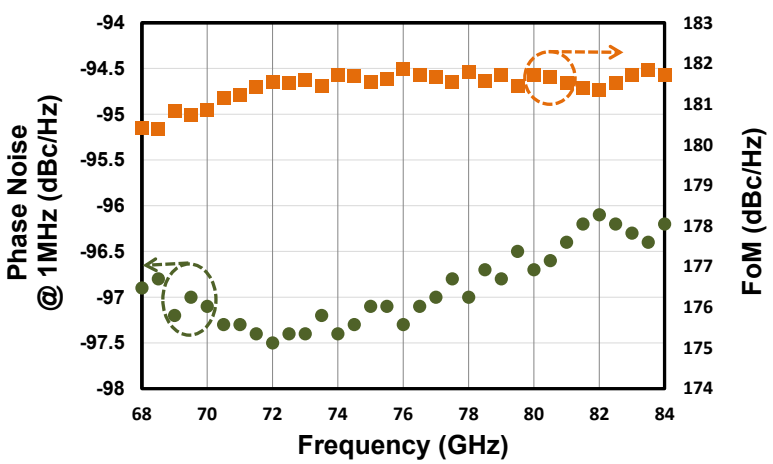

Fig. 9. Phase noise and figure-of-merit (FoM) at $1 \mathrm{MHz}$ offset versus E-band carrier frequency.

\section{REFERENCES}

[1] I. M. Milosavljevi et al., "A SiGe Highly Integrated FMCW Transmitter Module With a 59.5-70.5-GHz Single Sweep Cover," IEEE TMTT, vol. 66, no. 9, pp. 4121-4133, Sep. 2018.

[2] T. Siriburanon et al., "A 60-GHz Sub-Sampling Frequency Synthesizer Using Sub-harmonic Injection-Locked Quadrature Oscillators," in IEEE RFIC, 2014, pp. 105-108.

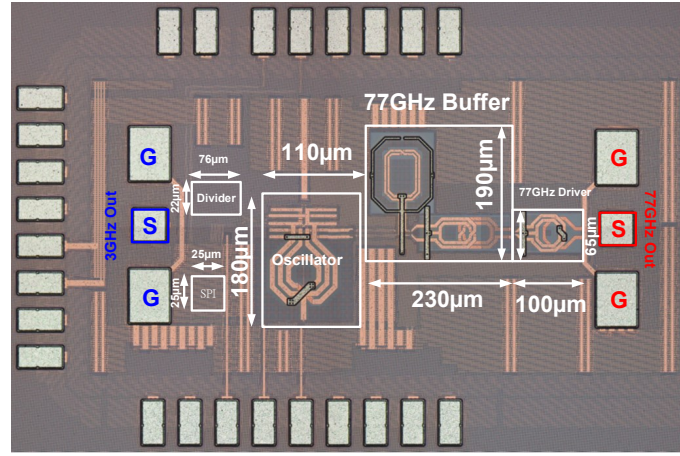

Fig. 10. Chip micrograph of the proposed $77 / 79 \mathrm{GHz}$ frequency generator.

[3] J. Lin et al., "A 77-GHz Mixed-Mode FMCW Signal Generator Based on Bang-Bang Phase Detector," IEEE JSSC, vol. 53, no. 10, pp. 28502863, Oct. 2018

[4] L. Wu, and Q. Xue, "E-Band Multi-PhaseLCOscillators WithRotatedPhase-Tuning UsingImplicit Phase Shifters," IEEE JSSC, vol. 53, no. 9 , pp. 2560-2571, Sep. 2018.

[5] J. Vovnoboy, R. Levinger, N. Mazor, and D. Elad, "A Dual-Loop Synthesizer With Fast Frequency Modulation Ability for $77 / 79 \mathrm{GHz}$ FMCW Automotive Radar Applications," IEEE JSSC, vol. 53, no. 5, pp. 1328-1337, May 2018.

[6] T.-N. Luo, C.-H. E. Wu, and Y.-J. E. Chen, "A 77-GHz CMOS FMCW Frequency Synthesizer With Reconfigurable Chirps," IEEE TMTT, vol. 61, no. 7, pp. 2641-2647, July 2013.

[7] J. Lee, Y.-A. Li, M.-H. Hung, and S.-J. Huang, "A Fully-Integrated 77GHz FMCW Radar Transceiver in 65-nm CMOS Technology," IEEE JSSC, vol. 45, no. 12, pp. 2746-2756, Dec. 2010.

[8] Z. Zong, M. Babaie, and R. B. Staszewski, "A $60 \mathrm{GHz}$ Frequency Generator Based on a $20 \mathrm{GHz}$ Oscillator and an Implicit Multiplier," IEEE JSSC, vol. 51, no. 5, pp. 1261-1273, May 2016.

[9] Z. Zong, P. Chen, and R. B. Staszewski, "A Low-Noise Fractional-N Digital Frequency Synthesizer with Implicit Frequency Tripling for mmwave Applications," IEEE JSSC, vol. 54, no. 3, pp. 755-767, Mar. 2019.

[10] S.-Y. Wu et al., "A 16nm FinFET CMOS Technology for Mobile SoC and Computing Applications," in IEEE IEDM, 2013, pp. 9.1.1-9.1.4. 